\title{
P-wave holographic superconductor/insulator phase transitions affected by dark matter sector
}

\author{
Marek Rogatko and Karol I. Wysokinski \\ Institute of Physics, Maria Curie-Sktodowska University, \\ 20-031 Lublin, pl. Marii Curie-Sktodowskiej 1, Poland \\ E-mail: rogat@kft.umcs.lublin.pl, karol@tytan.umcs.lublin.pl
}

ABSTRACT: The holographic approach to building the p-wave superconductors results in three different models: the Maxwell-vector, the SU(2) Yang-Mills and the helical. In the probe limit approximation, we analytically examine the properties of the first two models in the theory with dark matter sector. It turns out that the effect of dark matter on the Maxwell-vector p-wave model is the same as on the s-wave superconductor studied earlier. For the non-Abelian model we study the phase transitions between p-wave holographic insulator/superconductor and metal/superconductor. Studies of marginally stable modes in the theory under consideration allow us to determine features of p-wave holographic droplet in a constant magnetic field. The dependence of the superconducting transition temperature on the coupling constant $\alpha$ to the dark matter sector is affected by the dark matter density $\rho_{D}$. For $\rho_{D}>\rho$ the transition temperature is a decreasing function of $\alpha$. The critical chemical potential $\mu_{c}$ for the quantum phase transition between insulator and metal depends on the chemical potential of dark matter $\mu_{D}$ and for $\mu_{D}=0$ is a decreasing function of $\alpha$.

KEYwords: Holography and condensed matter physics (AdS/CMT), AdS-CFT Correspondence, Gauge-gravity correspondence, Black Holes

ARXIV EPRINT: 1508.02869 


\section{Contents}

1 Introduction $\quad 1$

2 Models of p-wave holographic superconductor with dark matter sector 3

2.1 Model of SU(2) Yang-Mills p-wave holographic superconductor with dark matter sector

3 P-wave holographic metal/superconductor phase transition

3.1 Condensation value

4 Insulator/holographic p-wave superconductor phase transition

4.1 Critical chemical potential

4.2 Critical phenomena

5 P-wave holographic droplet

6 Summary and conclusions

\section{Introduction}

The gauge/gravity duality provides a powerful theoretical method which enables a better understanding of the strongly coupled systems [1-3]. Originally proposed as the equivalence between type IIB superstring theory on $A d S_{5} \times S^{5}$ spacetime and $\mathcal{N}=4 \mathrm{SU}(N)$ supersymmetric Yang Mills theory on $(3+1)$-dimensional boundary, has later been generalized to other gravitational backgrounds [4]. The correspondence empowers an equality between the quantum field theory in $d$-dimensional spacetime and the gravity theory in $(d+1)$ dimensions, and its usefulness originates in a strong-weak duality [5]. Namely, the gravity dual of the strongly coupled quantum field theory is tractable in a perturbative approach.

The AdS/CFT correspondence has been recently proposed as a method to describe superconducting phase transition of the single s-wave superconductor [6]. Shortly afterwords it has been generalized to take into account other symmetries, like simple p or $\mathrm{d}$ wave. The generalizations require proper choice of the condensing field and appropriate gravity background. To describe d-wave holographic superconductor the charged massive spin-two field in the bulk [7-10] is required. The works related to building the holographic p-wave superconductor [11] have indicated a number of equally feasible possibilities and resulted in the multitude of approaches to study them. Inter alia the five-dimensional supergravity framework [13], and the Sturm-Liouiville eigenvalue problem [14] have been applied. A handful of novel results $[12,15,16]$ have also been reported. Especially intriguing result [15] is the change of the order of the superconducting transition showing up 
when backreaction is taken into account. The second order phase transition is replaced by the first order one, when matter field couplings are beyond a critical value.

Recently, the aforementioned studies were generalized in many other ways. The modification of gravity theory by considering the five-dimensional AdS solitonic metric has been proposed [17]. It enabled the construction of the holographic insulator/superconductor phase transition at zero temperature [18]. Namely, the AdS soliton line element dual to a confined field theory with a mass gap, imitates an insulator phase [19]. The strength of various kinds of matter backreactions has been shown to generate new phase transitions [20, 21].

The marginally stable modes of scalar and vector perturbations of the AdS spacetime have revealed the outset of the phase transition and help to study the influence of magnetic field on them $[22,23]$. In agreement with known phenomenology the magnetic field in the holographic theory makes the phase transition harder to occur. Both analytical and numerical methods devoted to the properties of s-wave and p-wave insulator/superconductor phase transitions were investigated in $[24,25]$. The studies in Gauss-Bonnet gravity were presented in $[26,30]$, the effects of the Weyl corrections on p-wave holographic phase transitions [27] were studied in [28]. The p-wave holographic superconductors in different gravity backgrounds [29], and in the presence of non-linear electrodynamics [31, 32] and other non-trivial conditions [33-35] have been elaborated.

The important problem being of special interest for the present work is a possible matter configuration in the Universe. According to numerous studies more than $24 \%$ of the matter is invisible and therefore dubbed as dark matter. There exist various proposals of how to model this component of matter. In this paper the point of view has been accepted according to which the dark matter is described by the U(1) field [21] analogous to the Maxwell one and coupled to the ordinary matter. The coupling constant $\alpha$ is treated as a free parameter, which value is bounded by $|\alpha|<2$.

This model of dark matter is supported by numerous astrophysical observations [3640] and other experimental data related to the muon anomalous magnetic moment [41] and experimental searching for the 'dark photon' [42-46].

The main aim of this paper is to study the influence of the dark matter sector on the properties of holographic p-wave phase transitions. These studies may hopefully result in discovery of some qualitatively untypical behavior which could be tested in the future experiments elucidating the cloven nature of dark matter.

The superconducting transition is signaled by spontaneous breaking of the U(1)-gauge symmetry. In the case under consideration the rotational symmetry is also broken by a special direction of a vector field, which is obtained by the condensation of a charged vector field. The present paper is the generalization of the previous works [47-49], were various aspects of phase transitions in s-wave holographic superconductor theory with ordinary matter sector coupled to the dark matter one have been elaborated. One expects that dark matter authorizes a part of a larger particle sector interacting with the visible matter and not completely decoupled [50-55].

The main point of our studies is the question how the dark matter sector modifies the ordinary phase transitions known from the previous studies of p-wave superconductors. The key role will be played by the dark matter coupling constant $\alpha$, binding dark matter 
fields with the ordinary Maxwell gauge field. It is important to know how the phase transitions are modified by the dark matter sector. To this end the holographic p-wave metal/superconductor and holographic insulator/p-wave superconductor phase transitions as well as p-wave holographic droplet embedded in magnetic field have been studied. As discussed in detail in section 2 the holographic p-wave superconductors provide a numerous non-trivial possibilities to look for the influence of the dark matter on them. Out of three possible models of holographic p-wave superconductors we shall discuss in detail two of them.

The paper is organized as follows. In section 2 we describe two of the three possible models used to build the p-wave symmetry superconductors and conclude that the effect of dark matter in one of them is formally the same as for s-wave symmetry. This prompts us to consider the other model for which this influence is much more interesting. In the subsection $\mathrm{B}$ we discuss the crucial points of the considered p-wave holographic superconductor model with the influence of the dark matter sector. In section 3, as a gravity background we assume five-dimensional AdS Schwarzschild line element and study the metal/superconductor phase transition. Dark matter sector effects on the insulator/holographic p-wave superconductor are analyzed in section 4 , while section 5 is devoted to p-wave holographic droplet in the presence of a constant magnetic field. We conclude our researches in section 6, paying attention to the new features of the elaborated phenomena induced by the presence of dark matter sector which can potentially serve as an indicator for the future experiments dedicated to the detection of dark matter.

\section{Models of p-wave holographic superconductor with dark matter sector}

In the literature on the subject there exist at least three different possible ways of building p-wave holographic superconductor. Namely, the Maxwell vector model [23], the SU(2) Yang-Mills $[11,15]$ one and the helical p-wave model $[56,57]$. The contemporary review of the subject can be found in [58]. Contrary to this, the quantum field theory approach (the weak coupling) delivers the unique description of p-wave superconductors, under the condition that one ought only to preserve the required symmetry. Namely, from the fact that superconductivity is related to a pairing of the two fermions it follows that the total wave function of the Cooper pair has to be antisymmetric, with respect to their exchanges. The antisymmetry of the spin part requires a symmetric orbital part of the wave function and one ends up with s-wave or d-wave superconductors. On the other hand, p-wave symmetry of the orbital part of the wave function requires the triplet character of the spin part. On the technical level, the field theoretical description of all types of superconductors stems from the same type of BCS-like equations which only differ by the symmetry of the form-factors, $g(\mathbf{k})=1$ for s-wave and $g(\mathbf{k})=k_{x}$, where $\mathbf{k}$ is a wave-vector, for the simplest p-wave symmetry. At present, it is not clear which of the aforementioned holographic models is the proper one for the description of strongly coupled p-wave superconductors and what are the differences between their properties. 
In this paper we shall study the Maxwell vector and the $\mathrm{SU}(2)$ Yang-Mills models. Our analysis relies on the theory in which the gravitational action is given by

$$
S_{g}=\int \sqrt{-g} d^{5} x(R-2 \Lambda),
$$

where $\Lambda=6 / L^{2}$ stands for the cosmological constant, while $L$ is the radius of the considered AdS spacetime.

Before we proceed to the main subject of the paper let us give some remarks about the other model of p-wave holographic superconductor, the so-called Maxwell vector model presented in [27], supplemented by the dark matter sector. The form of the action is in a close resemblance of the quantum electrodynamical $\rho$-meson, without irrelevant neutral part of it [59].

The gravitational part is the same as presented earlier, while the matter sector will be provided by the action

$$
\begin{aligned}
S_{m}=\int \sqrt{-g} d^{5} x( & -\frac{1}{4} F_{\mu \nu} F^{\mu \nu}-\frac{1}{4} B_{\mu \nu} B^{\mu \nu}-\frac{\alpha}{4} B_{\mu \nu} F^{\mu \nu} \\
& \left.-\frac{1}{2} \rho_{\mu \nu}^{\dagger} \rho^{\mu \nu}-m^{2} \rho_{\mu}^{\dagger} \rho^{\mu}+i q \gamma_{0} \rho_{\mu} \rho_{\nu}^{\dagger} F^{\mu \nu}\right),
\end{aligned}
$$

where a complex vector field $\rho_{\mu}$ with mass $m$ and the charge $q$ was introduced. $\rho_{\mu \nu}$ is defined by the covariant derivative $D_{\mu}=\nabla_{\mu}-i q A_{\mu}$ in the form given by

$$
\rho_{\mu \nu}=D_{\mu} \rho_{\nu}-D_{\nu} \rho_{\mu}
$$

The last term in equation (2.2) describes the magnetic moment of the vector field $\rho_{\mu}$. The vector field constitutes a charged U(1)-gauge field and on the AdS/CFT side is dual to an operator carrying the same charge under the symmetry in question. A vacuum expectation value of this operator will be subject to the spontaneous $U(1)$ symmetry breaking. The condensate of the dual operator breaks the U(1) symmetry and moreover because of the fact that we have to do with vector fields, the rotational symmetry is broken by choosing a special direction. In the light of the above claims, the vector field is treated as an order parameter and the model mimics p-wave superconductor.

Further, let us assume that we shall elaborate real vector field to have the connection with the results obtained in our previous studies. By the direct calculations it can be checked that, if we assume that the condensate picks out $x$-direction and $\mathrm{U}(1)$-gauge fields have only $t$-components

$$
\rho_{\alpha} d x^{\alpha}=\rho_{x} d x, \quad A_{\mu} d x^{\mu}=\phi(r) d t, \quad B_{\nu} d x^{\nu}=\eta(r) d t,
$$

the underlying Maxwell vector p-wave holographic superconductor with dark matter sector and with the real components of the vector field give us the same description of the phase transitions as the s-wave model studied earlier [49]. All the equations of motion are of the same forms when we exchange $\psi(r)$ (which acts as an order parameter in s-wave case) for the $\rho_{x}$-component of the vector field. Due to this fact we shall not elaborate the vector model and concentrate on the SU(2) Yang-Mills one in the following sections. 


\subsection{Model of SU(2) Yang-Mills p-wave holographic superconductor with dark matter sector}

In this section we shall describe the basic features of the SU(2) Yang-Mills holographic pwave superconductor model with the dark matter sector. To begin with one makes ansatz of an $\mathrm{SU}(2)$ Yang-Mills field and two U(1) subgroups of the $\mathrm{SU}(2)$, considered as the ordinary Maxwell one and the other supposed to describe the dark matter sector, coupled to the Maxwell electrodynamics. Next, a gauge boson generated by the other $\mathrm{SU}(2)$ generator and charged under U(1) Maxwell subgroup will be taken into account. On the other hand, the matter sector is provided by the action

$$
S_{m}=\int \sqrt{-g} d^{5} x\left(-\frac{1}{4} F_{\mu \nu}{ }^{(a)} F^{\mu \nu(a)}-\frac{1}{4} B_{\mu \nu}{ }^{(a)} B^{\mu \nu(a)}-\frac{\alpha}{4} B_{\mu \nu}{ }^{(a)} F^{\mu \nu(a)}\right),
$$

where $F_{\mu \nu}{ }^{(a)}$ and $B_{\mu \nu}{ }^{(a)}$ are two SU(2) Yang-Mills field strengths of the form $F_{\mu \nu}{ }^{(a)}=$ $\nabla_{\mu} A_{\nu}^{(a)}-\nabla_{\nu} A_{\mu}^{(a)}+\epsilon^{a b c} A_{\mu}^{(b)} A_{\nu}^{(c)}$. The totally antisymmetric tensor is set as $\epsilon^{123}=1$. The components of the gauge fields are bounded with the three generators of the $\mathrm{SU}(2)$ algebra by the relations $A=A_{\beta}^{(a)} \tau^{a} d x^{\beta}$, where $\left[\tau^{a}, \tau^{b}\right]=\epsilon^{a b c} \tau^{c}$. The parameter $\alpha$ describes the coupling between ordinary and dark matter $\mathrm{U}(1)$-gauge fields.

The equations of motion imply

$$
\nabla_{\mu} B^{\mu \nu(a)}+\frac{\alpha}{2} \nabla_{\mu} F^{\mu \nu(a)}+\epsilon^{a b c} B_{\mu}{ }^{(b)} B^{\mu \nu(c)}+\frac{\alpha}{2} \epsilon^{a b c} B_{\mu}{ }^{(b)} F^{\mu \nu(c)}=0 .
$$

and for $F_{\mu \nu}$ are provided by

$$
\nabla_{\mu} F^{\mu \nu(a)}+\frac{\alpha}{2} \nabla_{\mu} B^{\mu \nu(a)}+\epsilon^{a b c} A_{\mu}^{(b)} F^{\mu \nu(c)}+\frac{\alpha}{2} \epsilon^{a b c} A_{\mu}{ }^{(b)} B^{\mu \nu(c)}=0 .
$$

In order to simplify the above equations we multiply relation (2.6) by $\alpha / 2$ and extract the term $\frac{\alpha}{2} \nabla_{\mu} B^{\mu \nu(a)}$. The second term in the equation (2.7) is replaced by the aforementioned outcome. The final result may be written as

$$
\begin{aligned}
\tilde{\alpha} \nabla_{\mu} F^{\mu \nu(a)} & -\frac{\alpha}{2} \epsilon^{a b c} B_{\mu}^{(b)} B^{\mu \nu(c)}-\frac{\alpha^{2}}{4} \epsilon^{a b c} B_{\mu}{ }^{(b)} F^{\mu \nu(c)} \\
& +\epsilon^{a b c} A_{\mu}{ }^{(b)} F^{\mu \nu(c)}+\frac{\alpha}{2} \epsilon^{a b c} A_{\mu}{ }^{(b)} B^{\mu \nu(c)}=0
\end{aligned}
$$

where $\tilde{\alpha}=1-\frac{\alpha^{2}}{4}$.

Both SU(2) Yang-Mills fields, $A_{\mu}{ }^{(b)}$ and $B_{\mu}{ }^{(b)}$, are dual to some current operators in the four-dimensional boundary field theory. In order to accomplish a p-wave superconductor with dark matter sector we postulate that the following is satisfied

$$
\begin{aligned}
& A=\phi(r) \tau^{3} d t+w(r) \tau^{1} d x, \\
& B=\eta(r) \tau^{3} d t .
\end{aligned}
$$

In the above relations the $\mathrm{U}(1)$ subgroups of $\mathrm{SU}(2)$ group generated by $\tau^{3}$ are identified with the electromagnetic $\mathrm{U}(1)$-gauge field $(\phi(r))$ and the other $\mathrm{U}(1)$ group connected with the dark matter sector field $(\eta(r))$ coupled to the Maxwell one. The gauge boson field $(w(r))$ 
having the nonzero component along $x$-direction is charged under $A_{t}^{(3)}=\phi(r)$. According to the AdS/CFT dictionary, $\phi(r)$ is dual to the chemical potential on the boundary, whereas $w(r)$ is dual to $x$-component of a charged vector operator. The condensation of $w(r)$ field will spontaneously break the $\mathrm{U}(1)$ symmetry and is subject to the superconductor phase transition. It breaks the rotational symmetry by making the $x$-direction a special one and inclines the phase transition. The transition in question is interpreted as a p-wave superconducting phase transition on the boundary. As far as the U(1)-gauge field bounded with the dark matter sector is concerned, it has the component $B_{t}^{(3)}$ dual to a current operator on the boundary.

One can remark that the choice described by the relation (2.9) is the only consistent choice of the gauge field components allowing the analytic treatment of the problem. The direct calculations reveal that the $x(1)$ and $t(3)$ components of the equation (2.6) are given as follows:

$$
\begin{aligned}
\frac{\alpha}{2} \nabla_{\mu} F^{\mu x(1)}+\frac{\alpha}{2} \epsilon^{132} B_{t}^{(3)} F^{t x(2)} & =0 \\
\nabla_{\mu} B^{\mu t(3)}+\frac{\alpha}{2} \nabla_{\nu} F^{\nu t(3)} & =0
\end{aligned}
$$

while the same components of the equation (2.7) imply

$$
\begin{aligned}
\nabla_{\mu} F^{\mu x(1)}+\epsilon^{1 b c} A_{\mu}{ }^{(b)} F^{\mu x(c)} & =0, \\
\nabla_{\nu} F^{\nu t(3)}+\frac{\alpha}{2} \nabla_{\nu} B^{\nu t(3)}+\epsilon^{3 b c} A_{\mu}{ }^{(b)} F^{\mu t(c)} & =0,
\end{aligned}
$$

and the main relation (2.8) reduces to the following:

$$
\begin{aligned}
\tilde{\alpha} \nabla_{\mu} F^{\mu x(1)}-\frac{\alpha^{2}}{4} \epsilon^{132} B_{t}{ }^{(3)} F^{t x(2)}+\epsilon^{1 b c} A_{\mu}{ }^{(b)} F^{\mu x(c)} & =0, \\
\tilde{\alpha} \nabla_{\mu} F^{\mu t(3)}+\epsilon^{3 b c} A_{\mu}{ }^{(b)} F^{\mu t(c)} & =0 .
\end{aligned}
$$

We take into account the $x(1)$ and $t(3)$-components of the aforementioned equation.

\section{P-wave holographic metal/superconductor phase transition}

In order to analytically investigate metal/superconductor phase transition we shall implement the Sturm-Liouville method, which for the first time has been successfully used in holographic phase transition studies [60]. To commence with, one considers the background of five-dimensional black hole given by the line element

$$
d s^{2}=-g(r) d t^{2}+\frac{d r^{2}}{g(r)}+\frac{r^{2}}{L^{2}}\left(d x^{2}+d y^{2}+d z^{2}\right),
$$

where $g(r)=r^{2} / L^{2}-r_{+}^{4} / r^{2} L^{2}$. Without loss of generality we set $L=1$.

The Hawking temperature for the black hole in question, is equal to $T_{B H}=r_{+} / \pi$. The $t(3)$ and $x(1)$ components of the equation (2.8) are provided by the following relations:

$$
\partial_{r}^{2} \phi(r)+\frac{3}{r} \partial_{r} \phi(r)-\frac{w^{2}(r)}{\tilde{\alpha} r^{2} g} \phi(r)=0,
$$




$$
\partial_{r}^{2} w(r)+\left(\frac{1}{r}+\frac{\partial_{r} g}{g}\right) \partial_{r} w(r)+\frac{\phi(r) w(r)}{\tilde{\alpha} g^{2}}\left(\phi(r)-\frac{\alpha^{2}}{4} \eta(r)\right)=0 .
$$

The above set of the differential equations should be completed by the adequate boundary conditions. We impose that on the black hole event horizon $\phi\left(r_{+}\right)=0$ and the condensing field has a finite norm. The last condition requires that $w\left(r_{+}\right)$should also be finite. Consequently, the boundary conditions on the event horizon may be cast in the form

$$
\begin{aligned}
& \phi(r)=\phi_{\text {hor }}^{(1)}\left(1-\frac{r_{+}}{r}\right)+\ldots, \\
& w(r)=w_{\text {hor }}^{(0)}+w_{\text {hor }}^{(2)}\left(1-\frac{r_{+}}{r}\right)^{2}+\ldots
\end{aligned}
$$

On the other hand, on the boundary $r \rightarrow \infty$ of the considered spacetime one has

$$
\phi(r) \rightarrow \mu-\frac{\rho}{r^{2}}, \quad w(r) \rightarrow w^{(0)}+\frac{w^{(2)}}{r^{2}},
$$

where $\mu$ and $\rho$ are dual to the chemical potential and charge density, respectively. $w^{(0)}$ and $w^{(2)}$ are dual to the source and expectation value of the boundary vector operator. The requirement of having a normalizable solution implies that one sets the source $w^{(0)}$ equal to zero.

In $z=r_{+} / r$ - coordinates the equations of motion have the forms

$$
\begin{aligned}
\phi^{\prime \prime}(z)-\frac{1}{z} \phi^{\prime}(z)-\frac{w^{2}(z)}{\tilde{\alpha} z^{2} g_{0}} \phi(z) & =0, \\
w^{\prime \prime}(z)+\left(\frac{1}{z}+\frac{g_{0}^{\prime}}{g_{0}}\right) w^{\prime}(z)+\frac{r_{+}^{2} \phi(z)\left[\phi(z)-\frac{\alpha^{2}}{4} \eta(z)\right]}{\tilde{\alpha} z^{4} g_{0}^{2}} w(z) & =0,
\end{aligned}
$$

where' means derivative with respect to $z$-coordinate and $g_{0}=1 / z^{2}-z^{2}$.

For $T \rightarrow T_{c}$ the condensate is very small $w(z) \rightarrow 0$ and $w^{2}(z) \approx 0$. The value of the horizon radius for the black hole with temperature $T_{c}$ is denoted by $r_{+c}$. The equation (3.6) for the $\phi$ field reduces to the relation

$$
\phi^{\prime \prime}-\frac{\phi^{\prime}}{z} \simeq 0
$$

which has the general solution of the form $\phi(z)=c_{1}+c_{2} z^{2}$. Using the boundary conditions for it as described by the equations (3.3) and (3.5) we find $\phi \simeq \rho r_{+c}^{-2}\left(1-z^{2}\right)$. We need solution for the function $w(z)$ near the boundary $z \rightarrow 0$ of the considered spacetime. We shall seek it in the form

$$
\left.w(z)\right|_{z \rightarrow 0}=\langle\mathcal{R}\rangle z^{2} F(z),
$$

where we have assumed $w^{(0)}=0$ and denoted $\langle\mathcal{R}\rangle=w^{(2)}$.

To proceed further, we shall consider the equation (2.12) in order to find the relation between $\phi(z)$ and $\eta(z)$. It can be easily solved and one gets

$$
\eta(z)+\frac{\alpha}{2} \phi(z)=-\frac{c_{1}}{r_{+}^{4}} z^{4}+c_{2},
$$


where $c_{1}$ and $c_{2}$ are constants. This equation (for $\alpha=0$ ) shows that the asymptotic behavior of the field $\eta(z)$ has the form analogous to $\phi(z)$. This is correct as $\eta(z)$ is the counterpart of the field $\phi(z)$ in the dark sector. Due to the formal symmetry between both fields we assume the similar boundary conditions for $\eta(z)$. First, we require $\eta(1)=0$. It leads to the relation

$$
\eta(z)=\frac{c_{1}}{r_{+c}^{4}}\left(1-z^{4}\right)
$$

Comparing this dependence with the behavior of $\phi(z)$ close to the boundary $(z \rightarrow 0)$, we introduce the dark matter density $\rho_{D}=c_{1} / r_{+}^{2}$ and approximate the last equation close to $z=0$, by the following:

$$
\eta(z)=\frac{c_{1}}{r_{+c}^{4}}\left(1-z^{4}\right) \approx \frac{\rho_{D}}{r_{+c}^{2}}\left(1-z^{2}\right)
$$

This allows us to rewrite eq. (3.10) as

$$
\eta(z)=\frac{\rho_{D}}{r_{+c}^{2}}\left(1-z^{2}\right)-\frac{\alpha}{2} \phi(z) .
$$

Introducing the above relation into equation (3.7) and using representation (3.9) of $w(z)$ in terms of $F(z)$, we obtain the equation

$$
\left(p(z) F^{\prime}(z)\right)^{\prime}-q(z) F(z)+\Lambda^{2} r(z) F(z)=0,
$$

where we have set

$$
\begin{aligned}
& p(z)=z^{5} g_{0} \\
& q(z)=-z^{5} g_{0}\left(\frac{4}{z^{2}}+\frac{2}{z} \frac{g_{0}^{\prime}}{g_{0}}\right) \\
& r(z)=\frac{z\left(1-z^{2}\right)^{2}}{g_{0}}
\end{aligned}
$$

and denoted

$$
\Lambda^{2}=\rho^{2}\left[\tilde{\beta}-\frac{\alpha^{2}}{4} \frac{\rho_{D}}{\rho}\right] \frac{1}{\tilde{\alpha} r_{+c}^{6}},
$$

with $\tilde{\beta}$ given by the following expression:

$$
\tilde{\beta}=1+\frac{\alpha^{3}}{8} .
$$

The equation is solved by means of the Sturm-Liouville method with the function $F(z)$ satisfying the conditions $F(0)=1$ and $F^{\prime}(0)=0$. The Sturm-Liouville method enables us to find the minimum value of $\Lambda=\Lambda_{\text {min }}$ by minimizing the functional

$$
\Lambda^{2}=\frac{\int_{0}^{1} d z\left[F^{\prime}(z)^{2} p(z)+q(z) F^{2}(z)\right]}{\int_{0}^{1} d z r(z) F^{2}(z)} .
$$

The trial function has been chosen in the standard form $F(z)=1-a z^{2}$. The dependence of $g(r)$ on $r_{+}$has been taken into account by our definition of $g_{0}(z)=z^{-2}-z^{2}$ in the functions $p(z), q(z)$ and $r(z)$. 

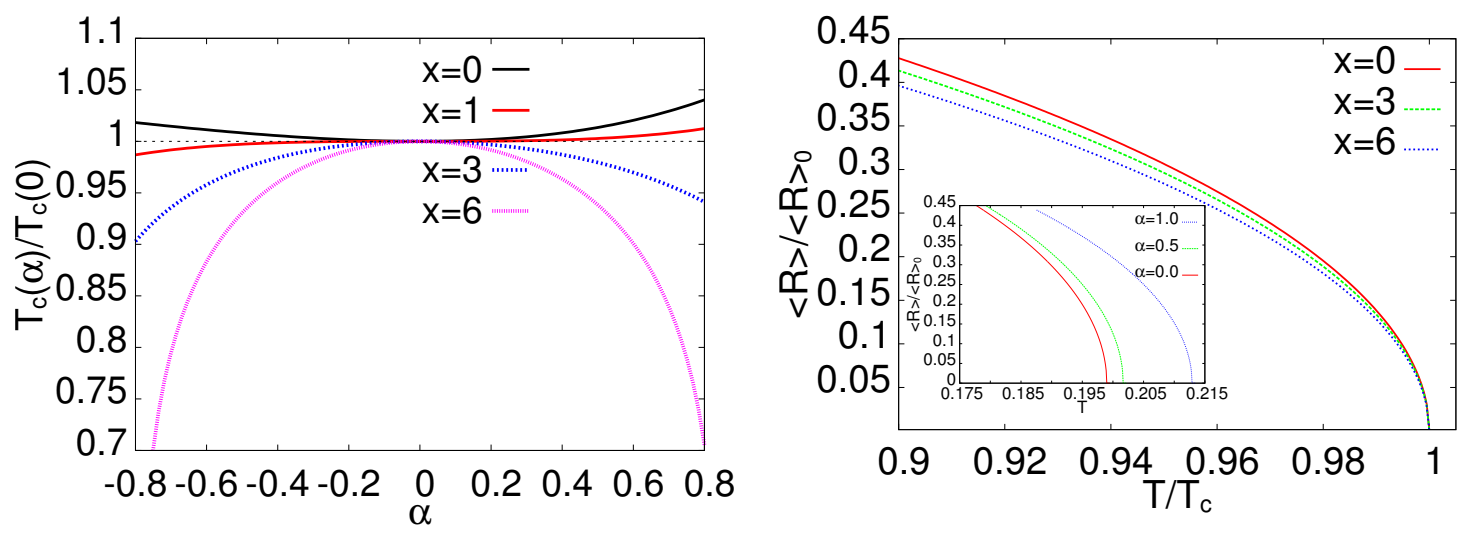

Figure 1. (color online) Left panel shows the $\alpha$ dependence of the transition temperature $T_{c}(\alpha) / T_{c}(0)$ normalized to its value without dark matter. The right panel depicts the dependence of the condensation value $\langle\mathcal{R}\rangle$ normalized to its value $\langle\mathcal{R}\rangle_{0}$ calculated for $\alpha=0$ on temperature $T$ normalized to the transition temperature $T_{c}$, for a few values of the ratio $x=\rho_{D} / \rho$ and $\alpha=0.5$. The inset shows the dependence $\langle\mathcal{R}\rangle /\langle\mathcal{R}\rangle_{0}$ on $T$ for $x=0$ and $\alpha=0,0.5,1.0$.

Having in mind that for a given black hole $T_{c}=r_{+c} / \pi$, it can be verified that the critical temperature is given by

$$
T_{c}(\alpha)=\frac{\rho^{\frac{1}{3}}}{\pi \Lambda_{\min }^{\frac{1}{3}}}\left(\frac{\tilde{\beta}-\frac{\alpha^{2}}{4} \frac{\rho_{D}}{\rho}}{\tilde{\alpha}}\right)^{\frac{1}{6}} .
$$

In the present model of p-wave metal/superconductor phase transition, we find that $T_{c}$ depends on the $\alpha$-coupling constant of dark matter sector even in the probe limit. This dependence reduces to the $(\tilde{\alpha} / \tilde{\beta})^{-1 / 6}$ form for $\rho_{D}=0$, which implies that the bigger $\alpha$ we take into account, the bigger value of the critical temperature one obtains. On the contrary, without backreaction, we have no such effect in s-wave holographic metal/superconductor phase transition in the theory with U(1)-gauge dark matter sector [48]. For general values of dark matter density the behavior is more complicated and is shown in figure 1 , for a few values of $\rho_{D}$. The value of $T_{c}(0)=\frac{\rho^{\frac{1}{3}}}{\pi \Lambda_{\min }^{\frac{1}{3}}}$ is the transition temperature of the considered superconductor in the absence of dark matter.

The previous experience from the study of dark matter sector effects in the backreacting s-wave holographic superconductor background [47], makes us to believe that the effects should be much stronger for p-wave symmetry superconductors, but this will be a subject of the future studies. Evaluating the Sturm-Liouville functional (3.20) we have found $\lambda_{\min } \approx 4.091$ for $a \approx 0.6845$. For $\rho=1$ one gets $T_{c}(0) \approx 0.199$. In the left panel of figure 1 , the dependence of the transition temperature on the coupling to the dark matter has been shown for a number of values $\rho_{D} / \rho$. We have normalized $T_{c}$ by dividing it by $T_{c}(0)$.

\subsection{Condensation value}

In this subsection, we shall pay attention to the influence of the dark matter sector on the condensation operator for the phase transition in question and its temperature dependence 
for $T$ not far from the critical one, $T_{c}$. One assumes a small but finite value of the condensate $\langle\mathcal{R}\rangle$ operator and writes

$$
\phi(z)=\frac{\rho}{r_{+c}^{2}}\left(1-z^{2}\right)+\langle\mathcal{R}\rangle \xi(z) .
$$

The function $\xi(z)$ corrects the function $\phi(z)$ evaluated at $T_{c}$ close to $z \approx 0$. At temperature $T$ the black hole is characterized by the horizon $r_{+}$such that $T=r_{+} / \pi$. The general behavior of the field $\phi(z)=\frac{\rho}{r_{+}^{2}}\left(1-z^{2}\right)$ is again deduced from eq. (3.5). Expanding $\xi(z)$ around $z=0$

$$
\xi(z)=\xi(0)+\xi^{\prime}(0) z+\frac{1}{2} \xi^{\prime \prime}(0) z^{2}+\ldots
$$

one deduces from $\phi(z=1)=0$ that $\xi(1)=0$. Comparing the coefficients multiplying the same powers of $z$ in the two expressions for $\phi(z)$ we get

$$
\begin{aligned}
\frac{\rho}{r_{+}^{2}} & =\frac{\rho}{r_{+c}^{2}}+\langle\mathcal{R}\rangle \xi(0) \\
\frac{\rho}{r_{+}^{2}} & =\frac{\rho}{r_{+c}^{2}}-\frac{1}{2}\langle\mathcal{R}\rangle \xi^{\prime \prime}(0) \\
\xi^{\prime}(0) & =0
\end{aligned}
$$

Consistency of the above equations requires $\xi(0)=-\xi^{\prime \prime}(0) / 2$. To find temperature dependence of $\langle\mathcal{R}\rangle$ we need the value of $\xi^{\prime \prime}(0)$.

In order to obtain $\xi^{\prime \prime}(0)$ we use the equation (3.6), introduce the expression (3.22) into it and get the relation for $\xi(z)$, valid up to the second order in $\mathcal{R}$

$$
\xi^{\prime \prime}-\frac{\xi^{\prime}}{z}=\frac{\rho\langle\mathcal{R}\rangle}{\tilde{\alpha} r_{+}^{2} r_{+c}^{2}} \frac{z^{2} F^{2}(z)\left(1-z^{2}\right)}{g_{0}} .
$$

It can be easily verified, by multiplying the above equation by $z$, that $\xi^{\prime}(0) \equiv 0$. It also provides that $\xi^{\prime \prime}(0)$ is finite. To find $\xi^{\prime \prime}(0)$ we rewrite left-hand side of the last equation as $\partial\left(\xi^{\prime}(z) / z\right) / \partial z$ and use the boundary conditions $\xi^{\prime}(1)=0$. It implies

$$
\xi^{\prime \prime}(0)=\left.\frac{\xi^{\prime}(z)}{z}\right|_{z \rightarrow 0}=-\frac{\lambda}{\tilde{\alpha}} \int_{0}^{1} d z \frac{z\left(1-z^{2}\right) F^{2}(z)}{g}=-\frac{\rho\langle\mathcal{R}\rangle}{2 \tilde{\alpha} r_{+}^{2} r_{+c}^{2}} E,
$$

where we set

$$
E=\int_{0}^{1} d z \frac{z^{3} F^{2}(z)}{\left(1+z^{2}\right)}
$$

Making use of eqs. (3.25) and (3.28) we find the dependence of the condensation value on temperature close to $T_{c}$

$$
\langle\mathcal{R}\rangle=\sqrt{\frac{2 \tilde{\alpha} \pi^{2}}{E}} T_{c}(\alpha) \sqrt{1+\frac{T}{T_{c}(\alpha)}} \sqrt{1-\frac{T}{T_{c}(\alpha)}} .
$$

Using the previously found value of $a=0.6845$, we obtain $E \approx 0.054046$ and temperature independent prefactor calculated for $\alpha=0$ is $\langle\mathcal{R}\rangle_{0} \approx 3.803$.

The right panel of figure 1 illustrates the dependence of the condensation operator $\langle\mathcal{R}\rangle$ normalized to its $\alpha=0$ value $\langle\mathcal{R}\rangle_{0}$ on temperature $T$, normalized to the actual transition 
temperature for three values of $x=\rho_{D} / \rho=0,3,6$ and $\alpha=0.5$. The condensation operator is a decreasing function of $x$, for a given value of $T / T_{c}(\alpha)$. The dependence of the condensation value on temperature for $x=0$ and three values of $\alpha=0,0.5,1$ is shown in the inset for $x=0$. The value of $\langle\mathcal{R}(T)\rangle$ decreases with the coupling $\alpha$ for constant ratio $T / T_{c}(\alpha)$ for all values of $x$. Its behavior as a function of $T$ is shown in the inset to the right panel of figure 1 for three values of $\alpha$.

The above equation envisages the fact that $\langle\mathcal{R}\rangle$ depends on $\alpha$-coupling constant of the dark matter sector directly via factor $\tilde{\alpha}^{1 / 2}$ and indirectly through $T_{c}(\alpha)$. It also depends on the density $\rho_{D}$ of the dark matter via $T_{c}$. The transition temperature increases with the coupling $|\alpha|$ for $\rho_{D}=0$. It features a monotonous increase with $\alpha$ for $x=1$ and strongly decreases with $|\alpha|$ for the value $\rho_{D} / \rho=6$, close to that inferred from astronomical observations. On the AdS/CFT side, the operator $\langle\mathcal{R}\rangle$ can be interpreted as responsible for the pairing mechanism. The smaller vacuum expectation value it has, the harder condensation happens. So we conclude that dark matter sector destructively influences the condensation phenomena in p-wave superconductors for $\rho_{D} / \rho>1$. As visible from figure 1 different behaviors are expected for $\rho_{D} / \rho=0.1$. The opposite (to that in the case $\rho_{D} / \rho>1$ ) conclusion was achieved in the case of s-wave holographic metal/superconductor phase transition [49]. Interestingly, in the Maxwell p-wave model we expect similar dependencies on dark matter sector as in s-wave superconductors. The observation of the effect in the laboratory could shed some light on the meaning of various models of holographic superconductors and their applicability for the description of real life materials. In the case of Gauss-Bonnet p-wave metal/superconductor phase transitions [61], the curvature corrections also imply the growth of the value of the condensation operator. This fact was also confirmed by the previous studies [62-64].

\section{Insulator/holographic p-wave superconductor phase transition}

The main ingredient in the considerations of insulator/superconductor phase transition will be the gravitational background of five-dimensional AdS soliton spacetime, given by

$$
d s^{2}=-r^{2} d t^{2}+L^{2} \frac{d r^{2}}{f(r)}+f(r) d \varphi^{2}+r^{2}\left(d x^{2}+d y^{2}\right)
$$

where $f(r)=r^{2}-r_{0}^{4} / r^{2}, r_{0}$ denotes the tip of the line element which constitutes a conical singularity of the considered solution. Without loss of generality we set the radius of the AdS spacetime equal to one. The AdS solitonic solution can be gained from the fivedimensional Schwarzschild-AdS black hole line element by making two Wick rotations. On the other hand, a conical singularity, at the tip $r=r_{0}$, can be get rid of by the ScherkSchwarz transformation of $\varphi$-coordinate in the form $\varphi \sim \varphi+\pi / r_{0}$. The gravitational background in question delivers the description of a three-dimensional field theory with a mass gap which in turn takes after an insulator in the condensed matter physics. The temperature of the aforementioned background equals to zero.

In order to solve the underlying equations of motion for the p-wave holographic insulator/superconductor phase transition problem, we shall impose the adequate boundary 
conditions. Namely, at the tip one has the same boundary conditions as in the s-wave insulator/superconductor problem

$$
\begin{gathered}
w=w_{0}+w_{1}\left(r-r_{0}\right)+w_{2}\left(r-r_{0}\right)^{2}+\ldots, \\
\phi=\phi_{0}+\phi_{1}\left(r-r_{0}\right)+\phi_{2}\left(r-r_{0}\right)^{2}+\ldots,
\end{gathered}
$$

where $w_{a}$ and $\phi_{a}$, for $a=0,1,2, \ldots$ are constants. One encumbers the Neumannlike boundary condition to obtain every physical quantity finite [18]. Contrary, near the boundary where $r \rightarrow \infty$, we have the different asymptotical behavior (comparing to the s-wave case). The asymptotic solutions read

$$
w \rightarrow w_{0}+\frac{w_{2}}{r^{2}}, \quad \phi \rightarrow \mu-\frac{\rho}{r^{2}},
$$

where $\mu$ and $\rho$ are interpreted as the chemical potential and the charge density in the dual theory, respectively. On the other hand, $w_{0}$ and $w_{2}$ have interpretations as a source and the expectation value of the dual operator. In order to gain the normalizable solution, one puts $w_{0}=0$ (we are not interested in the case when the dual operator is sourced).

In $z$-coordinates (with $r_{0}=1$ ) the equations in question yield

$$
\begin{aligned}
w^{\prime \prime}(z)+\left(\frac{f^{\prime}(z)}{f(z)}+\frac{1}{z}\right) w^{\prime}(z)+\frac{\phi(z)\left[\phi(z)-\frac{\alpha^{2}}{4} \eta(z)\right]}{\tilde{\alpha} f(z) z^{2}} w(z) & =0, \\
\phi^{\prime \prime}(z)+\left(\frac{f^{\prime}(z)}{f(z)}+\frac{1}{z}\right) \phi^{\prime}(z)-\frac{w^{2}(z)}{\tilde{\alpha} z^{2} f(z)} \phi(z) & =0 .
\end{aligned}
$$

\subsection{Critical chemical potential}

It was revealed numerically [18] that when the chemical potential exceeds its critical value the condensation operator turns on. In the case when $\mu<\mu_{c}$ the scalar field $w(r)$ is equal to zero, which is interpreted as the insulator phase. The system under consideration has a mass gap bounded with the confinement in $(2+1)$-gauge theory via the Scherk-Schwarz compactification. It justifies the conclusion that the critical value of the allowed potential is the turning point of the insulator/superconductor phase transition. Moreover, when $\mu \approx$ $\mu_{c}$, the scalar field $w(r)$ is so small that $w^{2}(r)=0$ and the equation (4.6) has an analytical solution in the form of a logarithmic function $\phi(z)=C_{1}+C_{2}\left[\log \left(1+z^{2}\right)-\log \left(1-z^{2}\right)\right]$ [23]. Establishing the value of the integration constant at the tip $z=1\left(C_{2}=0\right)$, one gains that $\phi(z)$ has the constant value $C_{1}=\mu$. From the boundary conditions, it can also be seen that near $z=0$ we get $\rho=0$.

As in the preceding section we find the dependence of $\phi(z)$ on the component of the dark matter sector $\eta(z)$. Using the the adequate components of the metric tensor for the line element (4.1) and equation (2.12), we arrive at the following relation

$$
\eta(z)+\frac{\alpha}{2} \phi(z)=D_{1}\left[\frac{z^{2}}{2}+\frac{1}{4} \log \left(\frac{1+z^{2}}{1-z^{2}}\right)\right]+D_{2},
$$

where $D_{1}$ and $D_{2}$ are integration constants. Setting the integration constant $D_{1}$ equal to zero at the tip $z=1$, we obtain

$$
D_{2}=\mu_{D}+\frac{\alpha}{2} \mu
$$


where $\mu_{D}$ denotes the chemical potential of the dark matter. For $\mu$ tending to its critical values $\mu_{c}$ from above, the equation of motion for $w(z)$ function is of the form

$$
w^{\prime \prime}(z)+\left(\frac{f^{\prime}(z)}{f(z)}+\frac{1}{z}\right) w^{\prime}(z)+\frac{\mu_{c}\left[\mu_{c}-\frac{\alpha^{2}}{4}\left(\mu_{D}-\frac{\alpha}{2} \mu_{c}\right)\right]}{f(z) z^{2} \tilde{\alpha}} w(z)=0 .
$$

To proceed we assume $w_{0}=$ and correct the solution for $w(z)$ close to the boundary $z=0$ by defining the trial function $G(z)$

$$
w(z) \sim\langle\mathcal{O}\rangle z^{2} G(z)
$$

with the boundary conditions $G(0)=1$ and $G^{\prime}(0)=0$ and $\langle\mathcal{O}\rangle=w_{2}$. For simplicity we set $r_{0}=1$. It implies further, that the equation (4.9) can be rewritten as

$$
\left(P(z) G^{\prime}(z)\right)^{\prime}-Q(z) G(z)+\tilde{\mu}^{2} R(z) F(z)=0,
$$

where we have defined the quantities as follows:

$$
\begin{aligned}
\tilde{\mu}^{2} & =\frac{\mu_{c}^{2}}{\tilde{\alpha}}\left(\tilde{\beta}-\frac{\alpha^{2}}{4} \frac{\mu_{D}}{\mu_{c}}\right), \\
P(z) & =z^{5} f, \\
Q(z) & =-f\left(4 z^{3}+2 z^{4} \frac{f^{\prime}}{f}\right), \\
R(z) & =z^{3} .
\end{aligned}
$$

The minimum eigenvalue of $\tilde{\mu}^{2}$ can be achieved from the variation of the functional

$$
\tilde{\mu}^{2}=\frac{\int_{0}^{1} d z\left[G^{\prime}(z)^{2} P(z)+Q(z) G^{2}(z)\right]}{\int_{0}^{1} d z R(z) G^{2}(z)} .
$$

With the choice of $G(z)=1-a z^{2}$ as the trial function we find $\tilde{\mu}_{\min } \approx 2.2666$ for $a \approx 0.3376$. Let's note that for $\alpha=0$ we have $\mu_{c}^{2}=\tilde{\mu}^{2}$ as it should be. The dependence of $\mu_{c}$ on $\alpha$ requires knowledge of $\mu_{D}$, which is unknown. For illustrational purposes we shall calculate the dependence $\mu(\alpha)$ for two values of $\mu_{D}=0, \mu_{c}$. In the first case we end up with the dependence $\mu=\tilde{\mu}_{\min } \sqrt{\tilde{\alpha} / \tilde{\beta}}$, while in the second case it has the form $\mu=\tilde{\mu}_{\min } \sqrt{\tilde{\alpha} /\left[\tilde{\beta}-\alpha^{2} / 4\right]}$.

The dependence of the critical chemical potential on $\alpha$ is shown in the left panel of figure 2 , for both values of $\mu_{D}$. For $\mu_{D}=0$ the chemical potential is the decreasing function of $\alpha$, independently of its sign. Contrary to that for $\mu_{D}=\mu_{c}$ the critical chemical potential increases for negative values of the coupling to dark matter, while decreases for positive $\alpha$. Thus, the transition appears at lower values of the chemical potential $\mu_{c}$ for positive values of $\alpha$, independently if $\mu_{D}$ vanishes or equals that of normal matter. This confirms the fact that dark matter makes the p-wave phase transition easier to happen provided the coupling is positive. Let's recall that for s-wave symmetry the holographic insulator/superconductor phase transition the chemical potential was unaffected by the presence of dark matter sector [49]. The result was similar as in Einstein-Maxwell theory [18, 24].

On the contrary, in the Gauss-Bonnet gravity the critical potential increases with the growth of the curvature corrections [26], making the condensation harder to form. This phenomenon was also found in s-wave holographic insulator/superconductor phase transition in Gauss-Bonnet system. 


\subsection{Critical phenomena}

When $\mu \rightarrow \mu_{c}$ the condensate value $\langle\mathcal{O}\rangle$ is small but finite and the equation of motion for the $\phi$ field is provided by

$$
\phi^{\prime \prime}+\left(\frac{f^{\prime}}{f}+\frac{1}{z}\right) \phi^{\prime}-\frac{\langle\mathcal{O}\rangle^{2} z^{2} F^{2}(z)}{\tilde{\alpha} f} \phi=0 .
$$

For $\mu$ slightly above the critical value the condensation scalar operator $\langle\mathcal{O}\rangle$ is very small. This enables us to look for the solution in the form

$$
\phi(z) \sim \mu_{c}+\langle\mathcal{O}\rangle \chi(z)+\ldots
$$

Moreover, to recover the previous result $\phi(z)=\mu$, one imposes the boundary condition $\chi(1)=0$ at the tip of the considered soliton. Near the boundary $z=0$, one expands the function $\chi(z)=\chi(0)+\chi^{\prime}(0) z+\frac{1}{2} \chi^{\prime \prime}(0) z^{2}+\ldots$, and rewrites $\phi$ in the form given by

$$
\phi(z) \simeq \mu-\rho z^{2} \simeq \mu_{c}+\langle\mathcal{O}\rangle\left(\chi(0)+\chi^{\prime}(0) z+\frac{1}{2} \chi^{\prime \prime}(0) z^{2}+\ldots\right),
$$

where we have imposed the boundary conditions at the tip of the soliton for the function $\chi(z)$ requiring that $\chi(1)=0$. Comparing the coefficients of the $z^{0}$-terms, in the above equation, we find that

$$
\mu-\mu_{c} \simeq\langle\mathcal{O}\rangle \chi(0) .
$$

Having in mind the relations (4.18) and (4.17), one can easily find the relation for $\chi(z)$. Namely, it is given by

$$
\chi^{\prime \prime}(z)+\left(\frac{f^{\prime}}{f}+\frac{1}{z}\right) \chi^{\prime}(z)-\frac{\langle\mathcal{O}\rangle \mu_{c} z^{2} F^{2}(z)}{\tilde{\alpha} f}+O\left(\langle\mathcal{O}\rangle^{n \geq 2}\right)=0
$$

where by $O\left(\langle\mathcal{O}\rangle^{n \geq 2}\right)$ we denoted terms of order $n \geq 2$ which can be neglected because of the fact that they are significantly smaller than the linear one. By virtue of the above we arrive at the relation

$$
\chi^{\prime \prime}(z)+\left(\frac{f^{\prime}}{f}+\frac{1}{z}\right) \chi^{\prime}(z)-\frac{\langle\mathcal{O}\rangle \mu_{c} z^{2} F^{2}(z)}{\tilde{\alpha} f} \simeq 0 .
$$

Let us redefine $\chi(z)$ function by the new one $\xi(z)$

$$
\chi(z)=\frac{\langle\mathcal{O}\rangle \mu_{c}}{\tilde{\alpha}} \xi(z) .
$$

The new definition of $\chi(z)$ allows to get rid of the $\tilde{\alpha}$-coupling dependence in the relation (4.22). One obtains

$$
\xi^{\prime \prime}+\left(\frac{f^{\prime}}{f}+\frac{1}{z}\right) \xi^{\prime}-\frac{z^{2} F^{2}(z)}{f}=0
$$

Consequently, the scalar operator $\langle\mathcal{O}\rangle$ provides the following:

$$
\langle\mathcal{O}\rangle=\sqrt{\frac{\left(\mu-\mu_{c}\right) \tilde{\alpha}}{\mu_{c} \xi(0)}},
$$


where $\xi(0)$ is given by

$$
\xi(0)=a_{1}-\int_{0}^{1} \frac{d z}{f z}\left(a_{2}+\int_{1}^{z} d y y^{3} F^{2}(y)\right) .
$$

The integration constants $a_{1}$ and $a_{2}$, are determined by the boundary conditions imposed on $\chi(z)$-function. To find them we have to fulfill the requirement $\xi(1)=0$. This leads to $a_{2}=0$ required to cancel the $a_{2} \log \left(1-z^{2}\right)$ term resulting from the integration over $z$ and $a_{1}=\left(3-2 a+2 a^{2}-4 a \log 2\right) / 24$. Using the previously found optimal value of $a=0.3375$ one ends up with $\xi(0)=a_{1} \approx 0.0894$.

On the other hand, the above relations reveal that the operator $\langle\mathcal{O}\rangle$ yields

$$
\langle\mathcal{O}\rangle \simeq \Delta\left(\mu-\mu_{c}\right)^{\frac{1}{2}}
$$

where the prefactor $\Delta=\sqrt{\frac{\tilde{\alpha}}{\mu_{c} \xi(0)}} \approx 3,139\left\{\tilde{\alpha}\left[\tilde{\beta}-\left(\alpha^{2} / 4\right)\left(\mu_{D} / \mu\right)\right]\right\}^{1 / 4}$ contains information on the dependence on dark matter sector. The previously found relation $\mu_{c}(\tilde{\alpha})=$ $\tilde{\mu} \sqrt{\tilde{\alpha} /\left[\tilde{\beta}-\left(\alpha^{2} / 4\right)\left(\mu_{D} / \mu\right)\right]}$ has to be taken into account.

Equation (4.27) envisages that the p-wave holographic/superconductor phase transition represents the second order phase transition for which the critical exponent has the mean field value $1 / 2$. This was also the case in s-wave insulator/superconductor phase transition influenced by the dark matter sector [48]. Thus the dark matter sector does not change the order of the transition.

The right panel of figure 2 illustrates the dependence of the condensation value $\langle\mathcal{O}\rangle$ on $\mu$ close to $\mu_{c}$. The dependence on $\mu / \mu_{c}(\alpha)$ shows that the amplitude of $\langle\mathcal{O}\rangle$ diminishes with $\alpha$. In fact the dark matter increases the value of the condensate operator $\langle\mathcal{O}\rangle$ if $\mu$ is kept constant as is visible from the inset. This conclusion is independent of the value of $\mu_{D}$ provided $\alpha>0$.

Next, we proceed to find the dependence of the charge density $\rho$ on the critical chemical potential. In order to calculate charge density $\rho$, we consider $z^{1}$-order coefficients in the relation (4.19), having in mind that $\xi^{\prime}(0)=0$, together with the previous requirement $\xi(1)=0$ being subject to the boundary condition. It leads to

$$
\chi^{\prime \prime}(0)=\left.\frac{\chi^{\prime}(z)}{z}\right|_{z \rightarrow 0}=-\langle\mathcal{O}\rangle \frac{\mu_{c}}{\tilde{\alpha}} \int_{0}^{1} d z z^{3} F^{2}(z)
$$

The comparison of the adequate coefficients of $z^{2}$-order in equation (4.19) leads to the conclusion that the charge density implies

$$
\rho=-\frac{\langle\mathcal{O}\rangle}{2} \chi^{\prime \prime}(0)
$$

By virtue of the equations (4.28) and (4.29), having in mind the relation (4.25), one arrives at the following

$$
\rho=\left(\mu-\mu_{c}\right) \tilde{D},
$$

where the quantity $\tilde{D}$ yields

$$
\tilde{D}=\frac{1}{2 \xi(0)} \int_{0}^{1} d z z^{3} F^{2}(z) .
$$

Evaluating the integral with the previously found parameters and introducing $\xi(0)$, we obtain $\tilde{D} \approx 0.844$. 

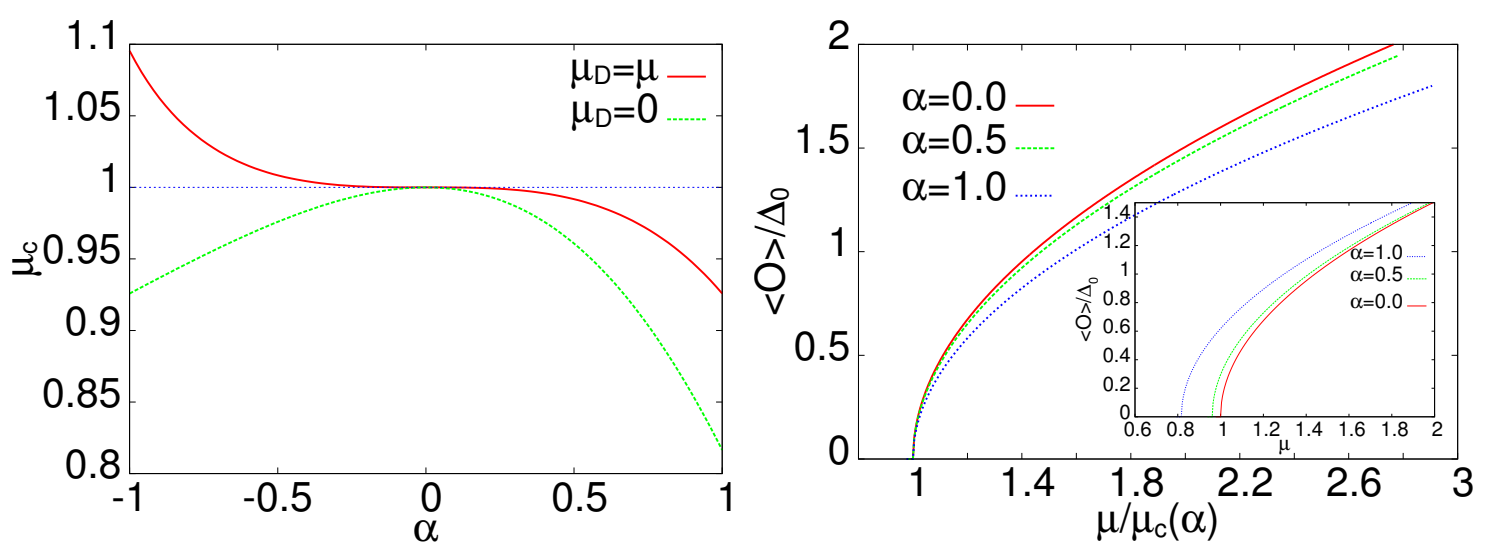

Figure 2. (color online) Left panel shows the dependence of the critical value of the chemical potential $\mu_{c}$, measured in units of $\tilde{\mu}$, for the insulator - holographic p-wave superconductor transition vs. $\alpha$ for two values of the dark matter chemical potential $\mu_{d}=0, \mu_{c}$. The dependence of the condensation value $\langle\mathcal{O}\rangle$ normalized to its $\alpha=0$ amplitude $\Delta_{0}$ on the normalized chemical potential $\mu / \mu_{c}(\alpha)$ is shown for $\mu_{D}=0$ and a few values of the coupling to the dark matter sector $\alpha=0,0.5,1.0$. The inset to the right panel shows similar dependence on the bar value of $\mu$, and for $\mu_{D}=0$.

\section{P-wave holographic droplet}

In this section, based on the notion of marginally stable modes of vector perturbations we shall investigate the influence of the magnetic field on holographic p-wave insulator/superconductor phase transition. We restrict the considerations to the probe limit.

To begin with, let us recall that the stability of a spacetime can be studied by the quasinormal modes (QNMs) of the perturbations in the background in question. When one encounters that the imaginary part of QNMs is negative, then they will decrease with the passage of time and disappear. The conclusion is that the elaborated spacetime is stable against these perturbations. On the other hand, the positivity of the imaginary part of QNMs reveals that the spacetime is unstable against the perturbations. The signal of instability or possible phase transition in the spacetime in question, is the occurrence of marginally stable modes $(\omega=0)$. They are only dependent on radial coordinates and do not backreact on the other fields.

As the gravitational background we shall use the line element of the five-dimensional AdS soliton, rewritten in the coordinates $(t, r, \tilde{\rho}, \varphi, \theta)$. It has the form

$$
d s^{2}=-r^{2} d t^{2}+\frac{d r^{2}}{f(r)}+f(r) d \varphi^{2}+r^{2}\left(d \tilde{\rho}^{2}+\tilde{\rho}^{2} d \theta^{2}\right)
$$

with $f(r)=r^{2}-r_{0}^{4} / r^{2}$. We assume the following components of the gauge fields $A_{\mu}^{(b)}$ and $B_{\mu}^{(b)}$ :

$$
\begin{aligned}
& A=\left(\mu_{c} d t+\frac{1}{2} B_{f} \tilde{\rho}^{2} d \theta\right) \tau^{3}+\psi(r, t, \varphi, \tilde{\rho}) \tau^{1} d \rho, \\
& B=\mu_{D} \tau^{3} d t
\end{aligned}
$$


where $B_{f}$ is the constant magnetic field. The time components of both fields $\mu_{c}$ and $\mu_{D}$ have constant values. This ansatz is valid close to the critical point of the phase transition. As in a previous section $\mu_{c}$ is the critical chemical potential for the phase transition in a droplet, and $\mu_{D}$ plays the role of the chemical potential of a dark matter.

The $\rho(1)$ component of the underlying equations of motion is provided by

$$
\partial_{r}^{2} \psi+\left(\frac{\partial_{r} f}{f}+\frac{1}{r}\right) \partial_{r} \psi+\frac{1}{f^{2}} \partial_{\varphi}^{2} \psi-\frac{1}{f} \partial_{t}^{2} \psi+\frac{1}{r^{2} f \tilde{\alpha}}\left[\mu_{c}\left(\mu_{c}-\frac{\alpha^{2}}{4} \mu_{D}\right)-\frac{B_{f}^{2} \tilde{\rho}^{2}}{4}\right] \psi=0
$$

In order to solve the above equation we choose an ansatz for $\psi$ field which implies

$$
\psi=F(r, t) H(\varphi) \mathrm{U}(\tilde{\rho}) .
$$

We set $\mathrm{U}(\tilde{\rho})=\tilde{\rho}=2 / \sqrt{B_{f}}$, because of the fact that one looks for the solution confined to a finite circular region which radius is proportional to $1 / \sqrt{B_{f}}$. Consequently, we arrive at the relations

$$
\begin{aligned}
\partial_{r}^{2} F+\left(\frac{1}{r}+\frac{\partial_{r} f}{f}\right) \partial_{r} F-\frac{r}{f} \partial_{t}^{2} F+\frac{1}{r^{2} f}\left(\frac{\mu_{c}\left(\mu_{c}-\frac{\alpha^{2}}{4} \mu_{D}\right)}{\tilde{\alpha}}-\frac{\lambda^{2} r^{2}}{f}-\frac{B_{f}}{\tilde{\alpha}}\right) F & =0 \\
\frac{\partial_{\varphi}^{2} H}{H} & =-\lambda^{2} .
\end{aligned}
$$

One considers the case when $\lambda=2 r_{0} n / L$, where $n \in Z$ causing the periodicity in $H(\varphi)=$ $H\left(\varphi+\pi L / r_{0}\right)$. In what follows without loss of the generality we set $r_{0}=1$ and $L=1$. Just, from periodicity property of $H(\varphi)$ we identify $\lambda=2 n$.

In the next step, one substitutes $F(r, t)=e^{-i \omega t} R(r)$. Consequently, the requirement concerning marginally stable modes leads to the condition that $\omega=0$. Next, the redefinition of the $r$-coordinates in terms of $z$ ones, provides the relation for $R(z)$

$$
\partial_{z}^{2} R(z)+\left(\frac{\partial_{z} f}{f}+\frac{1}{z}\right) \partial_{z} R(z)+\frac{1}{z^{2} f}\left(\frac{\mu_{c}\left(\mu_{c}-\frac{\alpha^{2}}{4} \mu_{D}\right)-B_{f}}{\tilde{\alpha}}-\frac{4 n^{2}}{z^{2} f}\right) R(z)=0 .
$$

Then, one introduces a trial function in the form

$$
\left.R(z)\right|_{z \rightarrow 0} \sim\langle\mathcal{O}\rangle z^{2} \Theta(z)
$$

with the boundary conditions imposed on $\Theta(z)$. Namely, $\Theta(0)=1$ and $\Theta^{\prime}(0)=0$.

After some algebra, the resulting equation can be converted into the standard SturmLiouville eigenvalue equation, which can be written as

$$
\partial_{z}\left(a(z) \Theta^{\prime}\right)-b_{n}(z) \Theta+\delta_{n}^{2} c(z) \Theta=0,
$$

where we have denoted by $\delta_{n}^{2}$ the relation

$$
\delta_{n}^{2}=\frac{\mu_{c}\left(\mu_{c}-\frac{\alpha^{2}}{4} \mu_{D}\right)-B_{f}}{\tilde{\alpha}} .
$$

The subscript $n$ is used to remind that the function $b(z)$ depends on the parameter $n$. 
The remaining quantities are defined by

$$
\begin{aligned}
a(z) & =f z^{5} \\
b_{n}(z) & =-f z^{4}\left(\frac{2}{z}+2\left(\frac{\partial_{z} f}{f}+\frac{1}{z}\right)-\frac{4 n^{2}}{z^{3} f^{2}}\right), \\
c(z) & =z^{3} .
\end{aligned}
$$

The eigenvalues of $\delta_{n}^{2}$ can be found by the method of minimizing the functional in question

$$
\delta^{2}=\frac{\mu_{c}\left(\mu_{c}-\frac{\alpha^{2}}{4} \mu_{D}\right)-B_{f}}{\tilde{\alpha}}=\frac{\int_{0}^{1} d z\left(\Theta^{\prime}(z)^{2} a(z)+b_{n}(z) \Theta(z)^{2}\right)}{\int_{0}^{1} d z c(z) \Theta^{2}(z)} .
$$

To minimize the above functional we have chosen as the trial function $B(z)=1-a z^{2}$. The Sturm - Liouville minimization gives $a \approx 0.942$ and $\delta^{2} \approx 3.8279$ for $n=1$, while one gets $a \approx 1.0083$ and $\delta^{2} \approx 5.6034$ for $n=2$. It can be seen that not only magnetic field influences the condensation but also $\alpha$ coupling constant of the dark matter sector as well as the component of the dark matter gauge field $B_{t}^{(3)}$, i.e., its chemical potential.

It can be noted that for $\mu_{D}=\mu_{c}$ the dependence on $\alpha$ cancels out. Having found $\delta_{n}^{2}$ we get the formula for $\mu_{c}$ the critical value of the chemical potential for metal-superconductor phase transition at temperature zero. Denoting the ratio of chemical potentials by $x=$ $\mu_{D} / \mu_{c}$, one gets

$$
\mu_{c}=\sqrt{\delta_{n}^{2}+B_{f}}\left(\frac{\tilde{\alpha}}{1-x \alpha^{2} / 4}\right)^{1 / 2} .
$$

The dependence of the chemical potential $\mu_{c}$ normalized by its $\alpha=0$ value equal to $\sqrt{\delta_{n}^{2}+B_{f}}$ on the coupling constant $\alpha$ is shown in the figure 3 , for three values of the dark matter chemical potential. For illustrational purposes we have chosen $\mu_{D}=0, \mu_{c}$ and $3 \mu_{c}$. For $\mu_{D}=0$ the critical value of the chemical potential $\mu_{c}$ is a decreasing function of $|\alpha|$. It takes on constant value for $x=1$ and is strongly increasing function of $|\alpha|$ for $x>1$.

\section{Summary and conclusions}

In the paper we have analytically investigated phase transitions towards p-wave holographic superconductors in which dar matter sector can imprints its presence. All the calculations were conducted in the probe limit, when the gravitational background was described by the five-dimensional AdS Schwarzschild black hole metric and AdS solitonic line element, respectively.

It was revealed that by a special choice of the real vector field, the vector Maxwell model of p-wave holographic superconductor is equivalent to s-wave description elaborated in [49]. All the properties of the considered phase transitions, like insulator/ superconductor and metal/superconductor phase transitions, in both models look identically. Of course, one should have in mind that the s-wave order parameter is replaced by $\rho_{x}$ component of the vector field, being in p-wave holographic superconductor an order parameter. Due to that fact we have considered in detail the $\mathrm{SU}(2)$ version of p-wave holographic superconductor. 


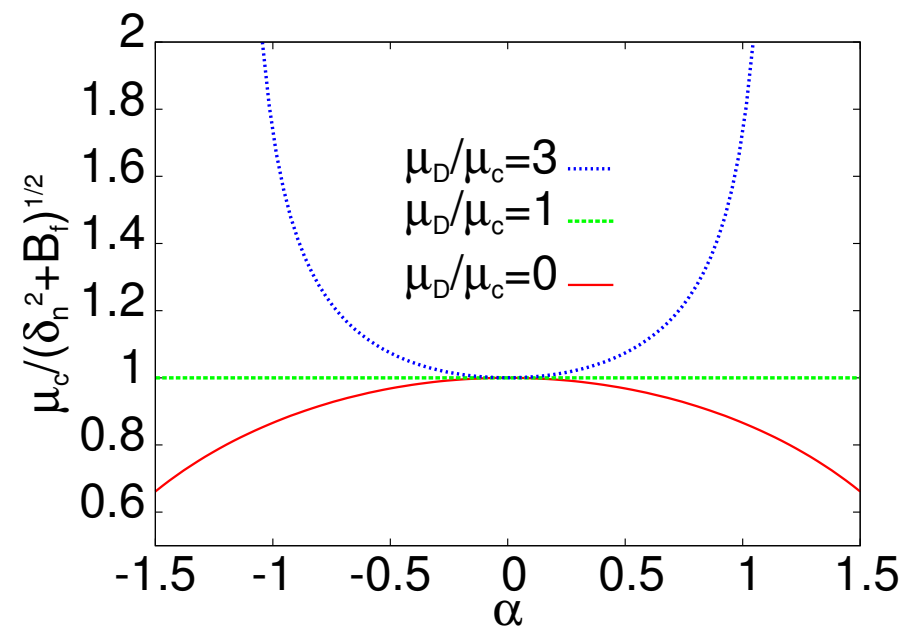

Figure 3. (color online). The dependence of the chemical potential $\mu_{c}$ normalized to $\sqrt{\delta_{n}^{2}+B_{f}}$ on the coupling constant $\alpha$ for the p-wave droplet for a few values of the parameter $x=\mu_{D} / \mu_{c}$.

Our ansatz for $\mathrm{SU}(2)$-gauge fields includes two U(1) groups, the subgroups of $\mathrm{SU}(2)$, one describing the ordinary Maxwell field and the other related to the dark matter sector.

Using the AdS/CFT approach and studying the properties of holographic superconductors in the background with dark matter sector we rely on the following three convictions. Firstly, we accept that the gauge-gravity duality teaches us about strongly coupled superconductors which are produced and studied in the laboratories. Some examples of this can be found in [65]. Secondly, the AdS/CFT correspondence relies on the AdS spacetime, while our space time is rather the dS one. The power of the gauge/gravity duality does not depend whether the Universe in which the superconductors exist, itself forms the AdS or de Sitter spacetime. It serves as a kind of calculus, which enables tackling strong coupling problems in a $d$ - dimensional field theory using perturbative approach in $d+1$ - dimensional gravity theory. Third, the dark matter existence seem to be obvious from astrophysical data as discussed in the Introduction. If it interacts with ordinary matter as proposed in the relation (2.5) and quantified by the coupling constant $\alpha$, so it modifies the behavior of the ordinary matter as visible from the equations (3.2). These modifications are found to influence the superconducting transition temperature and chemical potential and could, in principle, be observed as the annual changes of the properties of superconductors following the expected annual changes in the distribution of the dark matter [66].

Studies of the p-wave holographic metal/superconductor phase transitions reveal the critical temperature dependence on $\alpha$ - the coupling constant of the dark matter sector and other parameters of the dark matter: its chemical potential $\mu_{D}$ and its density $\rho_{D}$ - both unknown. So for illustrational purposes we have assumed that they may take arbitrary, albeit physically sensible values. The results depend on these values. For example it turns out that for $\rho_{D}=0$ the greater value of $\alpha$ one sets, the bigger value of the critical temperature one receives, cf. the left panel in figure 1. On the other hand for $\rho_{D} / \rho>1$ the increase of $\alpha$ results in decrease of $T_{c}$. This is an interesting result, which could potentially 
be of interest in possible experiments aimed at the detection of dark matter. However, one should remark that, the qualitatively similar dependence (as here for $\rho_{D}=0$ ) was earlier found in the case of the backreacting s-wave holographic superconductor in the theory under inspection [47]. It has to be checked to which extend the effect will be modified if the backreaction is taken into account in the model under consideration. The condensation value $\langle\mathcal{R}\rangle$ also exhibits dependence on the dark matter coupling constant.

As far as the holographic insulator/p-wave superconductor phase transition is concerned, it was spotted that the critical chemical potential also reveals the dependence on $\alpha$. For $x=0$ one observes the decrease of $\mu_{c}$ with $|\alpha|$ as shown in figure 2. In the case of Gauss-Bonnet gravitational background the situation is quite different. Namely, the critical potential increases with the development of the curvature corrections.

The determination of the condensation operator leads to the conclusion that p-wave holographic insulator/ superconductor phase transition is of the second order. The order parameter is proportional to $\left(\mu-\mu_{c}\right)^{1 / 2}$ with the mean field like exponent. Both $\mu_{c}$ and the prefactor depend on $\alpha$. Due to this fact, the condensation value $\langle\mathcal{O}\rangle$ may be increasing or decreasing function of $\alpha$ depending how one measures the distance from the critical chemical potential. If the distance is measured from the actual $\alpha$ dependent $\mu_{c}$ (main picture in the right panel of figure 2) $\langle\mathcal{O}\rangle$ is decreasing function of $\alpha$. On the other hand, for a given value of $\mu$, the increase of the coupling to dark matter appreciably enlarges the value of the condensation operator. On the other hand, the charge density is independent on $\alpha$-corrections except via $\mu_{c}$.

We have also elaborated p-wave holographic droplet in a constant magnetic field. In our studies we have used marginally stable modes of vector perturbations. It has been shown that the critical chemical potential of the superconducting droplet depends on the dark matter coupling $\alpha$, the magnetic field $B_{f}$ and dark matter chemical potential $\mu_{D}$. For $\mu_{D}=0, \mu_{c}$ diminishes as $|\alpha|$ grows. This is similar to the s-wave holographic droplet case with dark matter sector. For $\mu_{D}=\mu_{c}$ there is no dependence on $\alpha$, while the increase is observed for $\mu_{D}>\mu_{c}$.

It is worth mentioning that due to the fact that cosmic as well as on Earth experiments of detecting dark matter are in progress, the untypical behavior of SU(2) p-wave holographic superconductors during phase transitions in question may give a hint to unveil its secret.

\section{Acknowledgments}

We would like to thank the Referee for pointing out the inconsistency in our earlier choices of the fields $A_{\mu}$ and $B_{\mu}$. M.R. was partially supported by the grant of the National Science Center DEC-2013/09/B/ST2/03455 and KIW by the grant DEC-2014/13/B/ST3/04451.

Open Access. This article is distributed under the terms of the Creative Commons Attribution License (CC-BY 4.0), which permits any use, distribution and reproduction in any medium, provided the original author(s) and source are credited. 


\section{References}

[1] J.M. Maldacena, The large-N limit of superconformal field theories and supergravity, Int. J. Theor. Phys. 38 (1999) 1113 [hep-th/9711200] [INSPIRE].

[2] E. Witten, Anti-de Sitter space and holography, Adv. Theor. Math. Phys. 2 (1998) 253 [hep-th/9802150] [INSPIRE].

[3] S.S. Gubser, I.R. Klebanov and A.M. Polyakov, Gauge theory correlators from noncritical string theory, Phys. Lett. B 428 (1998) 105 [hep-th/9802109] [INSPIRE].

[4] J.P. Gauntlett, J. Sonner and T. Wiseman, Holographic superconductivity in M-theory, Phys. Rev. Lett. 103 (2009) 151601 [arXiv:0907.3796] [INSPIRE].

[5] S. Sachdev, What can gauge-gravity duality teach us about condensed matter physics?, Ann. Rev. Condensed Matter Phys. 3 (2012) 9 [arXiv:1108.1197] [INSPIRE].

[6] S.A. Hartnoll, C.P. Herzog and G.T. Horowitz, Building a holographic superconductor, Phys. Rev. Lett. 101 (2008) 031601 [arXiv:0803.3295] [INSPIRE].

[7] J.-W. Chen, Y.-J. Kao, D. Maity, W.-Y. Wen and C.-P. Yeh, Towards a holographic model of D-wave superconductors, Phys. Rev. D 81 (2010) 106008 [arXiv:1003.2991] [INSPIRE].

[8] F. Benini, C.P. Herzog, R. Rahman and A. Yarom, Gauge gravity duality for D-wave superconductors: prospects and challenges, JHEP 11 (2010) 137 [arXiv:1007.1981] [INSPIRE].

[9] M. Rogatko and K.I. Wysokiński, Remarks on the Hall conductivity in chiral superconductors: weak vs. strong coupling approach, Acta Phys. Polon. A 126 (2014) A9.

[10] H.-B. Zeng, Z.-Y. Fan and H.-S. Zong, D-wave holographic superconductor vortex lattice and non-Abelian holographic superconductor droplet, Phys. Rev. D 82 (2010) 126008 [arXiv:1007.4151] [INSPIRE].

[11] S.S. Gubser and S.S. Pufu, The gravity dual of a p-wave superconductor, JHEP 11 (2008) 033 [arXiv:0805.2960] [INSPIRE].

[12] P. Basu, J. He, A. Mukherjee and H.-H. Shieh, Hard-gapped holographic superconductors, Phys. Lett. B 689 (2010) 45 [arXiv:0911.4999] [InSPIRE].

[13] F. Aprile, D. Rodriguez-Gomez and J.G. Russo, p-wave holographic superconductors and five-dimensional gauged supergravity, JHEP 01 (2011) 056 [arXiv: 1011.2172] [INSPIRE].

[14] S. Gangopadhyay and D. Roychowdhury, Analytic study of properties of holographic p-wave superconductors, JHEP 08 (2012) 104 [arXiv:1207.5605] [INSPIRE].

[15] M. Ammon, J. Erdmenger, V. Grass, P. Kerner and A. O'Bannon, On holographic p-wave superfluids with back-reaction, Phys. Lett. B 686 (2010) 192 [arXiv:0912.3515] [INSPIRE].

[16] S. Liu and Y.-Q. Wang, Holographic model of hybrid and coexisting s-wave and p-wave Josephson junction, Eur. Phys. J. C 75 (2015) 493 [arXiv:1504.06918] [InSPIRE].

[17] G.T. Horowitz and R.C. Myers, The AdS/CFT correspondence and a new positive energy conjecture for general relativity, Phys. Rev. D 59 (1998) 026005 [hep-th/9808079] [INSPIRE].

[18] T. Nishioka, S. Ryu and T. Takayanagi, Holographic superconductor/insulator transition at zero temperature, JHEP 03 (2010) 131 [arXiv:0911.0962] [INSPIRE].

[19] E. Witten, Anti-de Sitter space, thermal phase transition and confinement in gauge theories, Adv. Theor. Math. Phys. 2 (1998) 505 [hep-th/9803131] [InSPIRE]. 
[20] G.T. Horowitz and B. Way, Complete phase diagrams for a holographic superconductor/insulator system, JHEP 11 (2010) 011 [arXiv:1007.3714] [INSPIRE].

[21] Y. Brihaye and B. Hartmann, Holographic superfluid/fluid/insulator phase transitions in $2+1$ dimensions, Phys. Rev. D 83 (2011) 126008 [arXiv:1101.5708] [InSPIRE].

[22] R.-G. Cai, X. He, H.-F. Li and H.-Q. Zhang, Phase transitions in AdS soliton spacetime through marginally stable modes, Phys. Rev. D 84 (2011) 046001 [arXiv:1105.5000] [INSPIRE].

[23] R.-G. Cai, L. Li, H.-Q. Zhang and Y.-L. Zhang, Magnetic field effect on the phase transition in AdS soliton spacetime, Phys. Rev. D 84 (2011) 126008 [arXiv:1109.5885] [InSPIRE].

[24] R.-G. Cai, H.-F. Li and H.-Q. Zhang, Analytical studies on holographic insulator/superconductor phase transitions, Phys. Rev. D 83 (2011) 126007 [arXiv:1103.5568] [INSPIRE].

[25] A. Akhavan and M. Alishahiha, p-wave holographic insulator/superconductor phase transition, Phys. Rev. D 83 (2011) 086003 [arXiv:1011.6158] [INSPIRE].

[26] Q. Pan, J. Jing and B. Wang, Analytical investigation of the phase transition between holographic insulator and superconductor in Gauss-Bonnet gravity, JHEP 11 (2011) 088 [arXiv: 1105.6153] [INSPIRE].

[27] R.-G. Cai, S. He, L. Li and L.-F. Li, A holographic study on vector condensate induced by a magnetic field, JHEP 12 (2013) 036 [arXiv: 1309. 2098] [INSPIRE].

[28] L. Zhang, Q. Pan and J. Jing, Holographic p-wave superconductor models with Weyl corrections, Phys. Lett. B 743 (2015) 104 [arXiv: 1502.05635] [INSPIRE].

[29] P. Chaturvedi and G. Sengupta, p-wave holographic superconductors from Born-Infeld black holes, JHEP 04 (2015) 001 [arXiv:1501.06998] [INSPIRE].

[30] R.-G. Cai, Z.-Y. Nie and H.-Q. Zhang, Holographic phase transitions of p-wave superconductors in Gauss-Bonnet gravity with back-reaction, Phys. Rev. D 83 (2011) 066013 [arXiv: 1012.5559] [INSPIRE].

[31] Z. Zhao, Q. Pan and J. Jing, Holographic insulator/superconductor phase transition with Weyl corrections, Phys. Lett. B 719 (2013) 440 [arXiv: 1212.3062] [INSPIRE].

[32] J. Jing, Q. Pan and S. Chen, Holographic superconductor/insulator transition with logarithmic electromagnetic field in Gauss-Bonnet gravity, Phys. Lett. B 716 (2012) 385 [arXiv: 1209.0893] [INSPIRE].

[33] T. Albash and C.V. Johnson, Vortex and droplet engineering in holographic superconductors, Phys. Rev. D 80 (2009) 126009 [arXiv:0906.1795] [InSPIRE].

[34] D. Roychowdhury, Holographic droplets in p-wave insulator/superconductor transition, JHEP 05 (2013) 162 [arXiv: 1304.6171] [inSPIRE].

[35] A. Amoretti, A. Braggio, N. Maggiore, N. Magnoli and D. Musso, Coexistence of two vector order parameters: a holographic model for ferromagnetic superconductivity, JHEP 01 (2014) 054 [arXiv: 1309.5093] [INSPIRE].

[36] P. Jean et al., Early SPI/INTEGRAL measurements of $511 \mathrm{keV}$ line emission from the $4^{\text {th }}$ quadrant of the galaxy, Astron. Astrophys. 407 (2003) L55 [astro-ph/0309484] [INSPIRE].

[37] J. Chang et al., An excess of cosmic ray electrons at energies of 300-800 GeV, Nature 456 (2008) 362 [INSPIRE]. 
[38] PAMELA collaboration, O. Adriani et al., An anomalous positron abundance in cosmic rays with energies 1.5-100 GeV, Nature 458 (2009) 607 [arXiv:0810.4995] [INSPIRE].

[39] D. Harvey, R. Massey, T. Kitching, A. Taylor and E. Tittley, The non-gravitational interactions of dark matter in colliding galaxy clusters, Science 347 (2015) 1462 [arXiv: 1503.07675] [INSPIRE].

[40] R. Massey et al., The behaviour of dark matter associated with four bright cluster galaxies in the $10 \mathrm{kpc}$ core of Abell 3827, Mon. Not. Roy. Astron. Soc. 449 (2015) 3393 [arXiv: 1504.03388] [INSPIRE].

[41] Muon G-2 collaboration, G.W. Bennett et al., Final report of the muon E821 anomalous magnetic moment measurement at BNL, Phys. Rev. D 73 (2006) 072003 [hep-ex/0602035] [INSPIRE].

[42] A. Afanasev et al., New experimental limit on photon hidden-sector paraphoton mixing, Phys. Lett. B 679 (2009) 317 [arXiv: 0810.4189] [INSPIRE].

[43] S.N. Gninenko and J. Redondo, On search for eV hidden sector photons in Super-Kamiokande and CAST experiments, Phys. Lett. B 664 (2008) 180 [arXiv:0804.3736] [INSPIRE].

[44] J. Suzuki, T. Horie, Y. Inoue and M. Minowa, Experimental search for hidden photon CDM in the eV mass range with a dish antenna, JCAP 09 (2015) 042 [arXiv:1504.00118] [INSPIRE].

[45] A. Mirizzi, J. Redondo and G. Sigl, Microwave background constraints on mixing of photons with hidden photons, JCAP 03 (2009) 026 [arXiv:0901.0014] [INSPIRE].

[46] J. Redondo and G. Raffelt, Solar constraints on hidden photons re-visited, JCAP 08 (2013) 034 [arXiv: 1305.2920] [INSPIRE].

[47] E. Nakonieczny and M. Rogatko, Analytic study on backreacting holographic superconductors with dark matter sector, Phys. Rev. D 90 (2014) 106004 [arXiv:1411.0798] [INSPIRE].

[48] Ł. Nakonieczny, M. Rogatko and K.I. Wysokiński, Magnetic field in holographic superconductor with dark matter sector, Phys. Rev. D 91 (2015) 046007 [arXiv:1502.02550] [INSPIRE].

[49] Ł. Nakonieczny, M. Rogatko and K.I. Wysokiński, Analytic investigation of holographic phase transitions influenced by dark matter sector, Phys. Rev. D 92 (2015) 066008 [arXiv: 1509.01769] [INSPIRE].

[50] T. Vachaspati and A. Achucarro, Semilocal cosmic strings, Phys. Rev. D 44 (1991) 3067 [INSPIRE].

[51] A. Achucarro and T. Vachaspati, Semilocal and electroweak strings, Phys. Rept. 327 (2000) 347 [Erratum ibid. 327 (2000) 427] [hep-ph/9904229] [INSPIRE].

[52] B. Hartmann and F. Arbabzadah, Cosmic strings interacting with dark strings, JHEP 07 (2009) 068 [arXiv: 0904.4591] [INSPIRE].

[53] Y. Brihaye and B. Hartmann, The effect of dark strings on semilocal strings, Phys. Rev. D 80 (2009) 123502 [arXiv:0907.3233] [INSPIRE].

[54] H. Davoudiasl, H.-S. Lee and W.J. Marciano, 'Dark' Z implications for parity violation, rare meson decays and Higgs physics, Phys. Rev. D 85 (2012) 115019 [arXiv:1203.2947] [INSPIRE]. 
[55] H. Davoudiasl, H.-S. Lee, I. Lewis and W.J. Marciano, Higgs decays as a window into the dark sector, Phys. Rev. D 88 (2013) 015022 [arXiv:1304.4935] [INSPIRE].

[56] A. Donos and J.P. Gauntlett, Holographic helical superconductors, JHEP 12 (2011) 091 [arXiv:1109.3866] [INSPIRE].

[57] A. Donos and J.P. Gauntlett, Helical superconducting black holes, Phys. Rev. Lett. 108 (2012) 211601 [arXiv: 1203.0533] [INSPIRE].

[58] R.-G. Cai, L. Li, L.-F. Li and R.-Q. Yang, Introduction to holographic superconductor models, Sci. China Phys. Mech. Astron. 58 (2015) 060401 [arXiv:1502.00437] [INSPIRE].

[59] D. Djukanovic, M.R. Schindler, J. Gegelia and S. Scherer, Quantum electrodynamics for vector mesons, Phys. Rev. Lett. 95 (2005) 012001 [hep-ph/0505180] [INSPIRE].

[60] G. Siopsis and J. Therrien, Analytic calculation of properties of holographic superconductors, JHEP 05 (2010) 013 [arXiv: 1003.4275] [INSPIRE].

[61] H.-F. Li, R.-G. Cai and H.-Q. Zhang, Analytical studies on holographic superconductors in Gauss-Bonnet gravity, JHEP 04 (2011) 028 [arXiv:1103.2833] [INSPIRE].

[62] R. Gregory, S. Kanno and J. Soda, Holographic superconductors with higher curvature corrections, JHEP 10 (2009) 010 [arXiv:0907.3203] [INSPIRE].

[63] R.-G. Cai, Z.-Y. Nie and H.-Q. Zhang, Holographic p-wave superconductors from Gauss-Bonnet gravity, Phys. Rev. D 82 (2010) 066007 [arXiv: 1007.3321] [INSPIRE].

[64] Q. Pan, B. Wang, E. Papantonopoulos, J. Oliveira and A.B. Pavan, Holographic superconductors with various condensates in Einstein-Gauss-Bonnet gravity, Phys. Rev. D 81 (2010) 106007 [arXiv:0912.2475] [INSPIRE].

[65] A.G. Green, An introduction to gauge gravity duality and its application in condensed matter, Contemp. Phys. 54 (2013) 33 [arXiv:1304.5908] [InSPIRE].

[66] K. Freese, M. Lisanti and C. Savage, Colloquium: annual modulation of dark matter, Rev. Mod. Phys. 85 (2013) 1561 [arXiv: 1209. 3339] [INSPIRE]. 